\title{
Speckle characterization in multimode fibers for sensing applications
}

\author{
Luis Rodriguez-Cobo, Mauro Lomer ${ }^{*}$, Carlos Galindez and J.M. Lopez-Higuera \\ Photonics Engineering Group, University of Cantabria, Av los Castros S/N, 39005, Santander, Spain
}

\begin{abstract}
This paper proposes the characterization of speckle patterns of multimode fibers in view of sensing applications and particularly for detection of vibration or seismic activity. Plastic optical fibers are used in this work due to its excellent flexibility and adaptability to build sensor heads. We are interested in the response to vibration, for which we use a short cylindrical piezoelectric transducer (PZT) vibrating in radial direction. The multimode fiber was coiled as tightly as possible around the mandrel of the PZT and periodic stretching effect was caused by the radial oscillations of the actuator. The PZT is modulated with a frequency generator by applying a sinusoidal signal in the range of 0 to $20 \mathrm{~Hz}$, so the speckle patterns can be time averaged. The fiber extreme is attached to a high speed camera with a plastic adaptor, centering the speckle pattern into the CCD. Maintaining the fiber position, a region of interest is selected to capture the video sequence and it is captured to detect the variations in the speckle pattern. Once having the video sequence, it is processed by averaging the pixel differences between two consecutive frames. This processed sequence is also filtered in order to reduce the high frequency noise component. In this work we report the results of the characterization of 3 types of multimode fibers, with core diameters of $50 \mu \mathrm{m}, 240 \mu \mathrm{m}$ and $980 \mu \mathrm{m}$.
\end{abstract}

Keywords: multimode fiber, speckle pattern, characterization, sensor application.

\section{INTRODUCTION}

Since optical fibers have been proposed as an alternative transmission medium to radio systems, there have been two types of fibers: single mode fibers and multimode fibers. In single mode fiber, the core diameter is small $(\approx 10 \mu \mathrm{m})$ and the optical signal has a phase velocity almost constant. In the multimode fiber, the diameter is much larger $(\geq 50 \mu \mathrm{m})$ and the guided modes have different phase velocities. In the first case, the projection of the beam at the output of the fiber, is a uniform spot of light, while in the second case it is observed a granulated pattern of light. The latter is the speckle pattern which is produced by an interference phenomenon between modes propagated inside of the fiber. These particular characteristics of the speckle phenomenon obtained in multimode fibers are used in sensing technology. In recent years several studies have been reported in vibration sensing [1], displacement [2], distance [3], cracks in concrete structures [4], and blood flow [5]. In all these cases, they have used multimode optical fibers with core diameters from 50 to $100 \mu \mathrm{m}$ and, usually, being glass fibers. Currently there are other multimode optical fibers, commercially available, and other materials such as Polymeric Optical Fiber (POF) with diameters of core $50 \mu \mathrm{m}$ to $3 \mathrm{~mm}$ [6-7]. The POF have numerous advantages in short-haul applications over glass.

This paper proposes the characterization of speckle patterns of multimode fibers in view of sensing applications and particularly for detection of vibration or seismic activity. POF are used in this work due to its excellent flexibility and adaptability to build sensor heads such as smaller curvature radius than the glass fiber or simplicity to connect. We are interested in the response to vibration, for which we use a short cylindrical piezoelectric transducer (PZT) vibrating in radial direction to characterize the POF response. The multimode fiber was coiled as tightly as possible around the mandrel of the PZT and periodic stretching effect was caused by the radial oscillations of the actuator. The PZT is modulated with a frequency generator by applying a sinusoidal signal in the range of 0 to $20 \mathrm{~Hz}$, so the speckle patterns can be time averaged. The POF extreme is attached to a 1394 high speed camera with a plastic adaptor, centering the speckle pattern into the CCD. Maintaining the POF position, a region of interest is selected to capture the video sequence and it is captured to detect the variations in the speckle pattern. Once having the video sequence, it is processed by averaging the pixel differences between two consecutive frames. This processed sequence is also filtered in order to reduce the high frequency noise component. In this work we report the results of the characterization of 3 types of multimode fibers, with core diameters of $50 \mu \mathrm{m}, 240 \mu \mathrm{m}$ and $980 \mu \mathrm{m}$.

\footnotetext{
*Email: lomerm@unican.Speckle 2012: V International Conference on Speckle Metrology, edited by

Ángel F. Doval, Cristina Trillo, J. Carlos López-Vázquez, Proc. of SPIE Vol. 8413, 84131R (C) 2012 SPIE · CCC code: $0277-786 X / 12 / \$ 18 \cdot$ doi: $10.1117 / 12.978217$
} 


\section{SPECKLE IN MULTIMODE FIBER}

Multimode fibers can spread a large number of modes with different phase velocities. The propagation modes corresponding to different optical paths used by the beams coupled into the fiber suffer different phase delays. The output field distribution consists of a sum of all individual contributions of each mode. If the contributions phase delay varies over $2 \pi$ radians, having a sufficiently coherent source then, the interference effects are well structured and can be observed in the intensity distribution through the end of the fiber. The number of modes, M, which supports an optical fiber break index is given by the expression [8] $M=V^{2} / 2$, where $V$ is called normalized frequency given by, $V=(2 \pi a / \lambda)\left[\left(n_{c o}\right)^{2}-\left(n_{c l}\right)^{2}\right]^{1 / 2}$, where $a$ is the core radius, $\lambda$ wavelength the laser and $n_{c o}$ and $n_{c l}$ are the refractive indexes of core and shell, respectively. For a gradient index fiber, the number of modes is: $M \approx V 2 / 4$. For example, if all modes are excited in the multimode fiber step-index, $n_{c o}=1.492$ and $n_{c l}=1.402$, and core radius $a=125$ and $490 \mu m$, the number of modes that would be about 200,000 and 3 million modes, respectively, when a He-Ne laser $(\lambda=0.6328 \mu m)$ is used. Due to the number of speckle dots present in a pattern is approximately equal to $\mathrm{M}$, a speckle pattern adaptable to the needs of the measured variable can be obtained just by choosing a fiber with a suitable diameter and consequently by determining the size of the speckle dots. Thus, when the conditions of the fiber change due to temperature, vibration or displacement, the speckle pattern is also modified, i.e., the phase velocities of the modes also change with time and with respect to the phase velocities of other modes. In practice, speckle pattern obtained in optical fibers is extremely sensitive to any environmental disturbance, showing a slow change even if it is affected by the above parameters. In this case, the pattern can be directly observed by projection of the light beam at the fiber output on a screen. But when the perturbations on the fiber are abrupt changes in the speckle pattern and they happen at a great speed, the origin of the cause just can be determined after a treatment of the signal captured by a camera. Figure 1 shows a photograph of speckle patterns at the output of multimode fibers POF with core diameters.
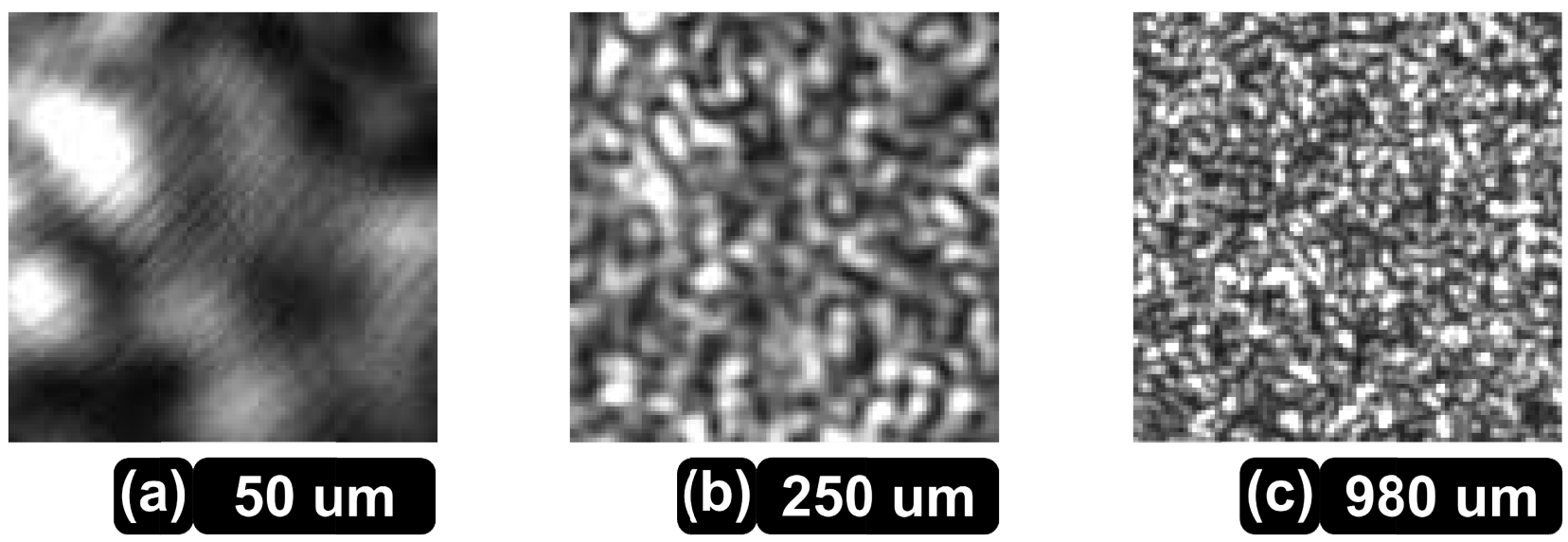

Figure 1. Speckle pattern produced by different core diameter of polymeric optical fibers, a) perfluorinated polymer a gradient index core diameter of $50 \mu \mathrm{m}, \mathrm{b}$ ) step index of $250 \mu \mathrm{m}$, and c) step index $980 \mu \mathrm{m}$.

\section{EXPERIMENTAL SETUP}

In our experiment, we have characterized three types of multimode POF, the characteristics of these fibers are listed in Table 1. The step-index POFs, with core diameter of $240 \mathrm{~mm}$ and $980 \mathrm{~mm}$, are of the same material and have the same numerical aperture. The graded-index POF core material is a polymer perfluorinated (PF), the maximum refractive index in the fiber axis, is 1.358 and the index of the cladding is 1.342 . For each of the experiences, 22 meters of fiber length have been used. In order to characterize the speckle pattern response of each fiber, a cylindrical PZT actuator of $40 \mathrm{~mm}$ of external diameter (manufactured by PI) is surrounded with the fiber under test with a single lap. To this multimode fiber to be measure, a He-Ne laser $(\lambda=0.6328 \mu \mathrm{m})$ is connected to the fiber in one end and the other (with the speckle pattern) is attached to a CCD high speed camera (Pixelink 74-10139). To get the deformation on the PZT external surface, a Fiber Bragg Grating (FBG) is also glued to have a deformation reference. The PZT actuator is modulated by applying a sinusoidal wave varying its frequency and amplitude from 1 to $25 \mathrm{~Hz}$ and from 500 and 1000 volts peak to peak respectively. The camera, the PZT actuator and the FBG interrogation unit (Micron Si425-500) are connected to a 
computer to control the experiment as shown on the Figure 2. A sweep of 6 steps on the amplitude range and with 5 different frequencies is made for each multimode fiber ( 3 x 30 different cases). For each modulation step, the modulation wave is maintained for 30 seconds to get an averaged value of the speckle value. The frame rate of the camera and the FBG interrogation unit is fixed to $250 \mathrm{~Hz}$, being the camera recording resolution limited to 96 by 96 pixels to reduce the amount of data. As it is shown on the Figure 1, there is still enough data for analyzing the speckle pattern without loosing resolution. Once the video sequences are captured the same processing method is employed for every case and it is shown on the Figure 3. A simple differential operation is applied between two consecutive frames, obtaining a single number related with the speckle pattern variation (solid line). A low pass filter is applied to the differential processed data to reduce the noise (dashed line) and the peaks of the sinusoidal wave are detected (maximums with diamonds and minimums with circles). For each situation an averaged value of the peak to peak is computed. The same peak to peak processing method is applied to the signal of the FBG to get the deformation reference.

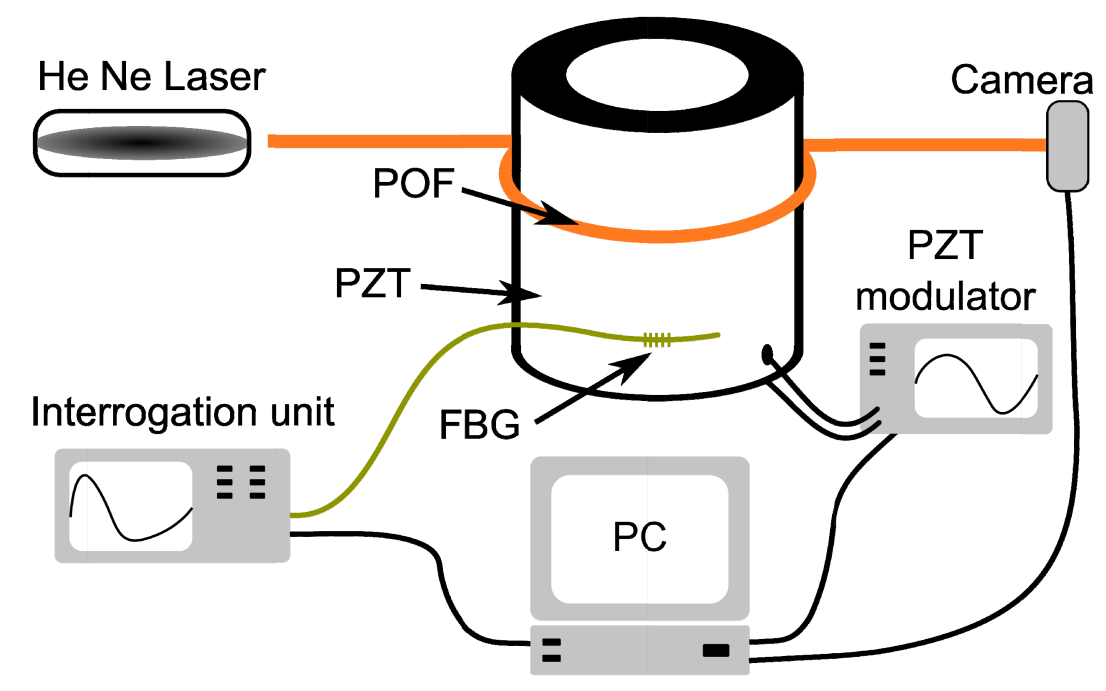

Figure 2. Schematic diagram of experimental characterization

Table 1. Characterized polymer optical fibers types [6-7]

\begin{tabular}{|c|c|c|c|c|c|}
\hline $\begin{array}{c}\text { Fiber ccre } \\
\text { material }\end{array}$ & $\begin{array}{c}\text { Core } \\
\text { diameter ( } \mu \mathrm{m})\end{array}$ & $\begin{array}{l}\text { Refractive } \\
\text { index }\left(n_{00}\right)\end{array}$ & $\begin{array}{l}\text { Numerical } \\
\text { aperture }\end{array}$ & $\begin{array}{l}\text { Weight } \\
(\mathrm{g} / \mathrm{m})\end{array}$ & $\begin{array}{l}\text { Number } \\
\text { modes }\end{array}$ \\
\hline PMMA (step-index) & 980 & 1.49 & 0.5 & 1.0 & $3^{\prime} 082,691$ \\
\hline PMNA (step-index) & 240 & 1.49 & 0.5 & 0.01 & $1 \quad 184,884$ \\
\hline Perfluoninated (graded-index) & 50 & $(1.342-1.358)$ & 0.2 & - & 665 \\
\hline
\end{tabular}

${ }^{8} \lambda=0.6328 \mu \mathrm{m}$ 


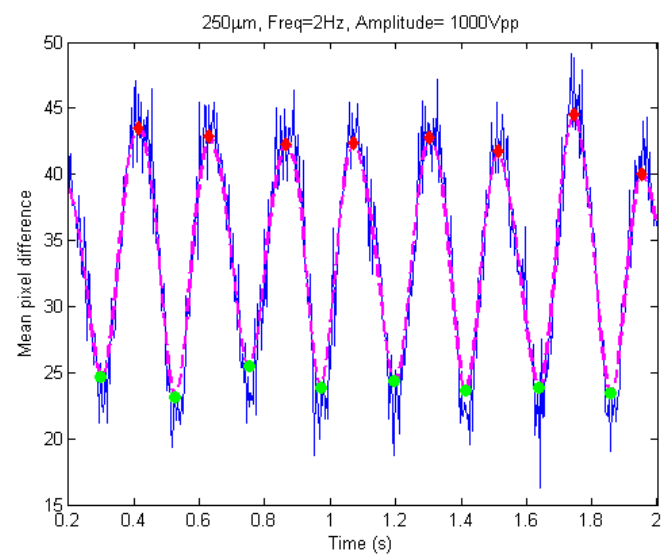

Figure 3. Example of the speckle processed data. The differential process of the video sequence causes a signal proportional to the speckle pattern variation (solid line). This signal is low pass filtered (dashed line) and the peak are detected (diamonds and circles) to get the averaged peak to peak ratio for each case.

\section{RESULTS AND DISCUSSION}

The deformation of the PZT actuator is proportional to the control voltage. The obtained data with the 3 multimode fibers is plotted against the deformation measured by the FBG on the Figure 4. There are shown each of the 6 PZT voltage steps causing a specific peak to peak deformation measured at $25 \mathrm{~Hz}$ for being the most sensitive case. Due to the proposed differential processing scheme, the setup is most sensitive for higher frequencies but always far from the camera sampling rate. The two tested fibers with a higher number of modes have a very good linear response with the deformation, having a $R^{2}$ coefficient above 0.99 at the best case with the higher tested frequency. On the contrary, the less sensitive tested fiber ( $50 \mathrm{um}$ ) fits worse the linear response even for the best case.

According to the Figure 1, the amount of contained light granules in the speckle pattern is function of the core diameter of the multimode fiber. The larger core diameter, the greater the amount of speckle, but the smaller the size of speckle. The sensitivity of the speckle pattern to changes by the vibrations effect can be caused by the amount and size of the speckle generated by multimode fibers. If all the fibers are characterized in the same conditions and a factor to be considered is the mass of the fiber. In fact, Fig. 4 shows that among the three characterized fibers, multimode fiber diameter of $240 \mu \mathrm{m}$ is more sensitive to variations of the vibration. One explanation for this result may be due to the difference in weight compared with the fiber of $980 \mu \mathrm{m}$ diameter. As shown in Table 1, the fiber of $240 \mu \mathrm{m}$ has a mass 100 times less than the fiber of $980 \mu \mathrm{m}$, so it is much lighter and more sensitive. Low responsiveness of the fiber diameter of $50 \mu \mathrm{m}$ may be due to the lesser amount of modes and the size of each speckle.

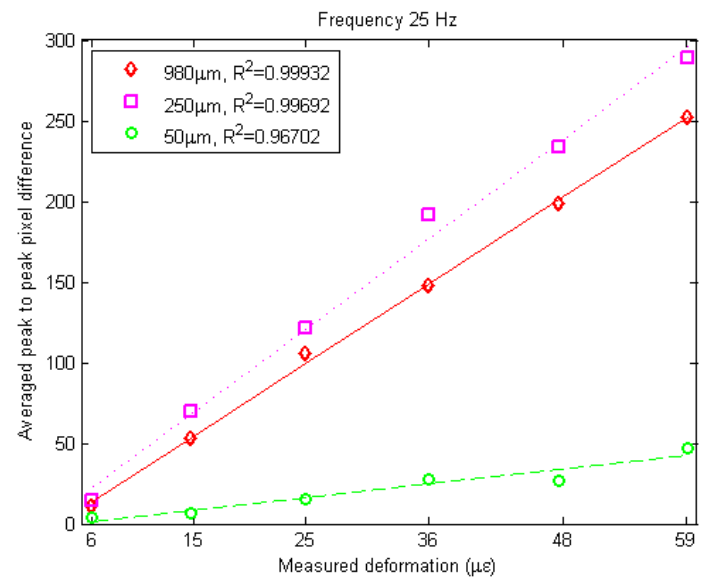

Figure 4. Response of the 3 multimode fibers against the PZT peak to peak deformation at $25 \mathrm{~Hz}$. 

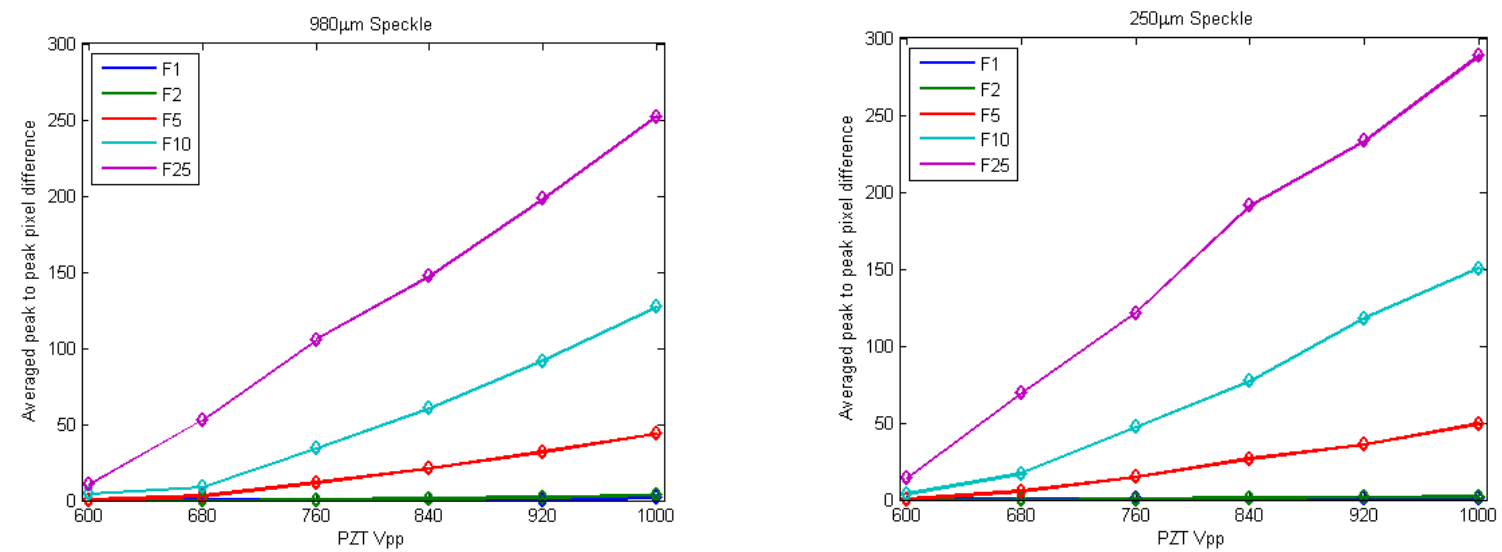

Figure 5. Response of the 2 multimode fibers with a higher number of modes $980 \mu \mathrm{m}$ (left) and $240 \mu \mathrm{m}$ (right). As the PZT modulation frequency is increased the sensitivity of the setup also increased.

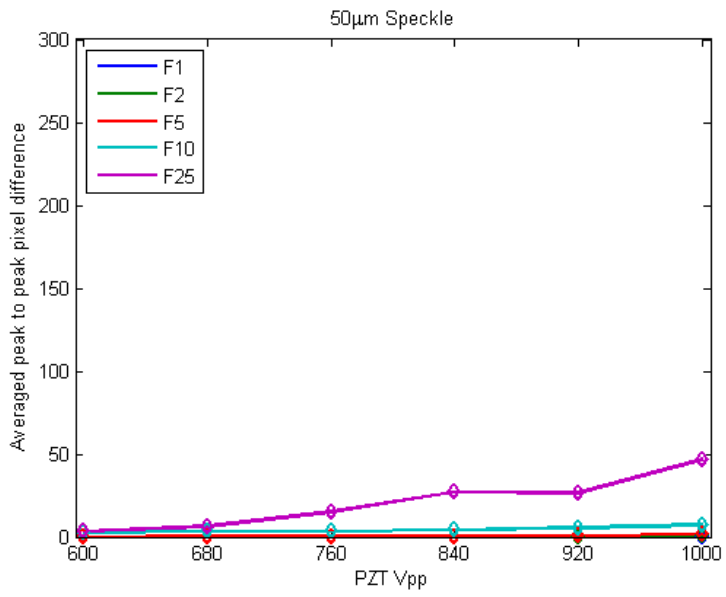

Figure 6. Response of the multimode fiber with less number of modes 50um. The overall response of the setup is poor in comparison with the other tested fibers.

In Figure 5 and 6 there are plotted the mean value of the pixel difference depending on the voltage applied to the PZT for different frequencies of $1 \mathrm{~Hz}, 2 \mathrm{~Hz}, 5 \mathrm{~Hz}, 10 \mathrm{~Hz}$ and $25 \mathrm{~Hz}$. Using the same scale, it can be seen that the sensitivity of the speckle pattern increases linearly with the voltage applied to the PZT and the number of modes. But also influences the fiber mass. When the fiber diameter decreases, the size of the speckle is getting larger, so the variations are smaller and smaller (in the case of Figure 6). In the latter case it may be useful to measure vibrations in compact structures such as buildings.

As an application example of the multimode fiber speckle pattern and the described signal processing method, the Figure 7 is included. On the Figure 7 (a) there is shown the speckle processed sequence captured from a POF attached to a cylindrical PZT that is modulated with a sinusoidal wave. On the Fig. 1 (b) it is also shown the representation of a human heart beating measured by surrounding a finger with a loop of POF. The sequence is filtered with a low pas filter in order to isolate the heart signal. In this case we used a GI-POF of $50 \mu \mathrm{m}$ diameter. 


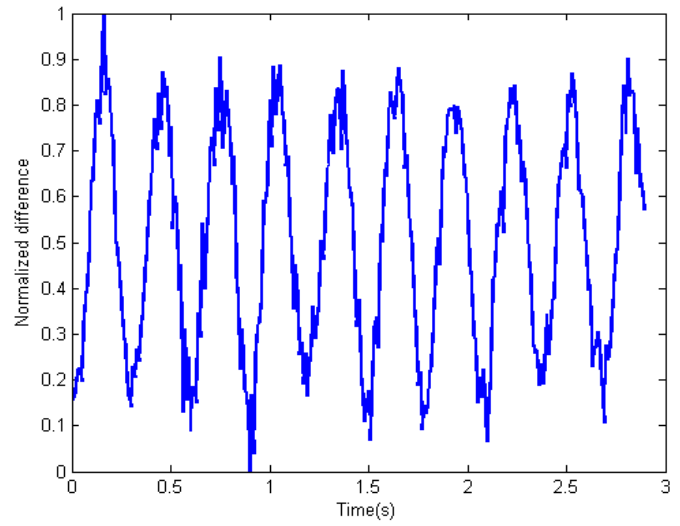

(a)

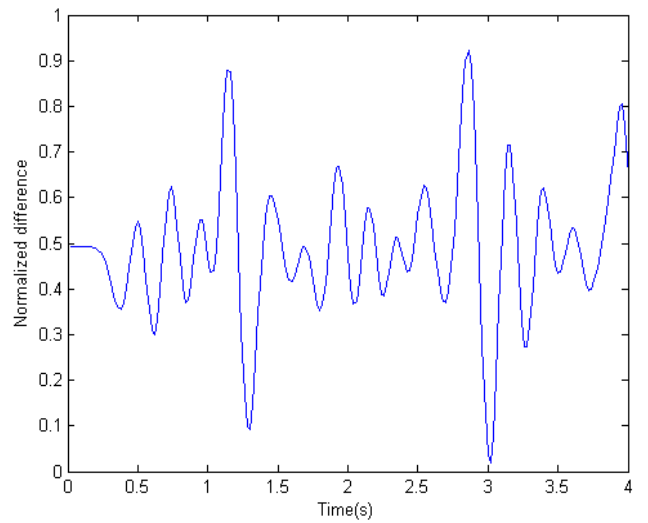

(b)

Figure 7. Results obtained on a multimode fiber with gradient index of $50 \mu$ m core diameter.

\section{CONCLUSION}

It has been performed the characterization of the speckle pattern using several types of multimode optical fibers. The speckle pattern has been subjected to vibration by a PZT and an FBG interrogation unit in the frequency range from 1 to $25 \mathrm{~Hz}$. The influence of the size and number of speckle dots in function of frequency has also been studied.

The method of processing speckle signal captured by the CCD camera is simple and can be integrated in a sensing system. To obtain the speckle pattern using multimode fibers introduces numerous advantages in sensor systems due to the versatility of the fibers. They are easy to use, have flexibility and low cost and particularly, polymer optical fibers are easy to handle.

\section{ACKNOWLEDGMENTS}

The authors acknowledge the Spanish government for supporting this work through the TEC2010-20224-CO2-02 projects and with the grant AP2009-1403.

\section{REFERENCES}

[1] Spillman, W.B.Jr., Kline, B.R., Maurice, L.B., and Fuhr, P.L., "Statistical-mode sensor for fiber optic vibration sensing uses", Applied Optics, 28, 3166-3176, (1989).

[2] Yu, F.T.S., Wen, M., Jing, S., and Uang, C.M., "Submicrometer displacement sensing using inner-product multimode fiber speckle fields", Applied Optics, 32, 4685-4689, (1993).

[3] Kitagawa, Y., and Hayashi, A., "Fiber-optic sensor for distance and velocity measurements using speckle dynamics", Applied Optics, 24, 955-959, (1985).

[4] Zhang, Z., and Ansari, F., "Fiber-optic laser speckle-intensity crack sensor for embedment in concrete", Sensors and Actuators A-126, 107-111, (2006).

[5] Dietsche, G., Ninck, M., Ortolf, C., Li, J., Jaillon, F., and Gisler, T., "Fiber-based multispeckle detection for timeresolved diffusing-wave spectroscopy: characterization and application to blood flow detection in deep tissue", Applied Optics, 46, 8506-8514, (2007).

[6] Mitsubishi Rayon Co., Ltd., http://www.pofeska.com/pofeskae/product/01/index.html.

[7] Chromis Fiberoptics, Inc, http://www.chromisfiber.com/techno why.htm

[8] Goodman, J.W., Speckle Phenomena in Optics, Roberts and Company, Chapter 7, Englewood, Colorado, (2007). 